\title{
p38MAPK Signaling Pathway in Osteoarthritis: Pathological and Therapeutic Aspects
}

\author{
Zongchao Li', Aonan Dai', Ming Yang', Siyu Chen ${ }^{2,3}$, Zhenhan Deng $\mathbb{D}^{2,3, *}$, Liangjun Li',* \\ 'Department of Orthopaedics, The Affiliated Changsha Central Hospital, Hengyang Medical School, University of South China, Changsha, People's \\ Republic of China; ${ }^{2}$ Department of Sports Medicine, The First Affiliated Hospital of Shenzhen University, Shenzhen Second People's Hospital, \\ Shenzhen, People's Republic of China; ${ }^{3}$ School of Clinical Medicine, Guangxi University of Chinese Medicine, Nanning, People's Republic of China
}

*These authors contributed equally to this work

Correspondence: Zhenhan Deng, Department of Sports Medicine, The First Affiliated Hospital of Shenzhen University, Shenzhen Second People's Hospital, 3002 Sungang West Road, Shenzhen City, 5I 8035, People's Republic of China, Tel +86 I3928440786, Fax +86 755-83366388, Email dengzhenhan@email.szu.edu.cn; Liangjun Li, Department of Orthopaedics, The Affiliated Changsha Central Hospital, Hengyang Medical School, University of South China, I6I Shaoshan South Road, Changsha City, 4I00I8, People's Republic of China, Tel +86 I3875822004, Fax+86731-85668I56, Email lilianngjun1212@sina.com

\begin{abstract}
Osteoarthritis (OA) is an aging-related joint disease, pathologically featured with degenerated articular cartilage and deformation of subchondral bone. OA has become the fourth major cause of disability in the world, imposing a huge economic burden. At present, the pathogenesis and pathophysiology of OA are still unclear. Complex regulating networks containing different biochemical signaling pathways are involved in OA pathogenesis and progression. The p38MAPK signaling pathway is a member of the MAPK signaling pathway family, which participates in the induction of cellular senescence, the differentiation of chondrocytes, the synthesis of matrix metalloproteinase (MMPs) and the production of pro-inflammatory factors. In recent years, studies on the regulating role of p38MAPK signaling pathway and the application of its inhibitors have attracted growing attention, with an increasing number of in vivo and in vitro studies. One interesting finding is that the inhibition of p38MAPK could suppress chondrocyte inflammation and ameliorate OA, indicating its therapeutic role in OA treatment. Based on this, we reviewed the mechanisms of p38MAPK signaling pathway in the pathogenesis of OA, hoping to provide new ideas for future research and OA treatment.
\end{abstract}

Keywords: osteoarthritis, p38MAPK signaling pathway, aging, inflammation

\section{Introduction}

Osteoarthritis (OA) is a leading cause of joint pain and disability, bringing huge socioeconomic costs in the global context. ${ }^{1,2}$ There are approximately 300 million individuals worldwide who are suffering from $\mathrm{OA}^{3} \mathrm{OA}$ is characterized by progressive degeneration of the articular cartilage (AC) and ligaments, as well as chronic synovitis and abnormal bone remodeling. Knee and hip joints are the most commonly affected synovial joints. ${ }^{4}$ With the progression of OA, patients will suffer from increasingly severe pain, limited autonomic activity, and even disability. Recent studies indicate that the progression of OA is affected by multiple factors, such as aging, gender, genetics, obesity, etc. ${ }^{5}$ However, most of the current therapies for OA, including the oral administration of non-steroidal anti-inflammatory drugs (NSAIDs) and glucosamine, are limited to symptom relief and pain management. Disease-modifying drugs which can reverse the progression of OA are still not available. ${ }^{6}$ Orthopedic surgeries, typically total joint replacement surgeries, remain the last choice for OA treatment. ${ }^{7}$ Although orthopedic surgeries are able to improve the quality of life to some extent, potential complications such as pain, opioid abuse, infection, and poorer joint function must be taken into consideration when opting for surgical intervention. ${ }^{8,9}$ Thus, finding new therapeutic targets for the development of disease-modifying treatments is of great importance.

The mitogen-activated protein kinase (MAPK) is a kind of serine/threonine protein kinase widely existing in eukaryotic cells. It can be activated by multiple internal and external stimulators, such as extracellular signals, physical 
stimulation, bacteria, tumor growth factor (TGF) and inflammatory cytokines like interleukin (IL)-1 and IL-6. After being activated, MAPK transfers the extracellular signal to the nucleus and regulates the activity of transcription factors, which will eventually alter the expression of related genes and induce cell reaction. ${ }^{10}$ Existing research suggests that the MAPK signaling pathway and OA progression are internally related. The dysregulation of the MAPK signaling pathway will accelerate the inflammatory response, leading to the release of massive cartilage matrix degrading enzymes and aggravation of cartilage degeneration. ${ }^{10}$ Therefore, MAPK is deemed to play an important role in the progression of $\mathrm{OA}$ and is currently considered as a major signaling pathway in the regulation of OA. ${ }^{11-14}$ The subfamilies of MAPK include p38MAPK, extracellular regulated protein kinases (ERKs), and c-Jun N-terminal kinase (JNK). ${ }^{9}$ To date, MAPK and the receptor tyrosine kinases, including ERKs, JNK and p38MAPKs have been confirmed to be involved in the pathogenesis of OA. ${ }^{12-15}$ This paper aimed to review the regulatory effects of the p38MAPK signaling pathway in the pathogenesis of OA, hoping to provide new targets for future OA treatment.

\section{Characteristics of p38MAPK Signaling Pathway}

The p38MAPK signaling pathway is a member of the MAPK super family (see Figure 1 for the basic information of p38MAPK pathway). It was discovered by Brewster et $\mathrm{al}^{16}$ in 1993 when they were studying fungi under the hypertonic environment. In 1994, Han et $\mathrm{al}^{17}$ first cloned p38MAPK from the mouse liver cDNA library and found that it was tyrosinephosphorylated in response to the endotoxic lipopolysaccharide (LPS). There are four subtypes of P38, namely P38 $\alpha$, P38 $\beta$, $\mathrm{P} 38 \gamma$ and P38 . More specifically, P38 $\alpha$ and P38 $\beta$ can be further divided into two isomers P38 $\alpha 1 / \alpha 2$ and P38 $\beta 1 / \beta 2 .{ }^{18}$ Each subtype has its own genetic code and is distributed in different tissues: $\mathrm{P} 38 \alpha$ is widely present in various tissues; $\mathrm{P} 38 \beta$ is the most abundant subtype in the brain tissue; P38 $\gamma$ mainly exists in the skeletal muscle; and P38 is commonly found in testes, pancreas, prostate, small intestine, etc. ${ }^{19}$ The amino acid sequences of all homologous kinases are highly similar that all kinases contain the "Thr-XAA-Tyr" tripeptide sequence and their homology is over $50 \%{ }^{20}$

The p38MAPK pathway transmits extracellular signals into cells through a cascade reaction involving multilevel kinases. MAPKK3 and MAPKK6 are the main activators of p38MAPK. ${ }^{21}$ Three key kinases are required to be activated sequentially to induce the MAPK cascade reaction: ${ }^{22}$ MAPKKK (TAK, MLK3 and ASK), MAPKK (MKK3 and MKK6), and MAPK. A variety of extracellular stimuli can activate the p38MAPK pathway in articular chondrocytes and induce phosphorylation cascades. These stimuli include but are not limited to inflammatory factors and cytokines in the articular fluid, changes of osmotic pressure, changes of biological stress and rays. ${ }^{23-25}$ The activation of MAPKKK is followed by the phosphorylation of the double sites of Thr (tyrosine) and Tyr (threonine) on the "T-ring structure" of p38MAPK, which is executed by MKK3/ MKK6. Then, p38MAPK will be activated subsequently. The activated p38MAPK can either enter the nucleus or migrate to other sites, and then activate the transcription factors (eg, ATF2/6, MAX, HSF-1, CHOP/GADD153, myocyte enhancement factor-2, nuclear transcription factor $\beta$, AT, H-1/2, ETS-1, ELK-1, and SAP-1) accordingly. ${ }^{26}$

Recent studies have provided ample evidence, supporting that the p38MAPK signaling pathway is associated with a variety of age-related diseases. ${ }^{27-32}$ For example, according to Moreno-Cugnon et al, the elevated p38MAPK activity was associated with neural stem cell aging. ${ }^{29}$ Chen et al suggested that RAB7 was a protective factor for intervertebral discs, which retarded the intervertebral disc degeneration by inhibiting the p38MAPK pathway. ${ }^{31}$ In the report published by Kheiri et al, p38MAPK was revealed to play a role in the progression of Alzheimer's disease, and its inhibitors delayed or even reversed the progression of the disease. ${ }^{32}$ As a degenerative joint disease closely related to aging process, OA has been found regulated by p38MAPK in several key aspects (Figure 2).

\section{Key Biomolecules Involved in p38MAPK-Regulated OA Progression MMPs and ADAMTS}

$\mathrm{OA}$ is a common senile degenerative joint disease affecting all kinds of tissues of the whole joint, including cartilage, subchondral bone, synovial membrane, and infrapatellar fat pad. ${ }^{1}$ The main pathological features of OA are chondrocyte degeneration and extracellular matrix (ECM) disintegration. ${ }^{2,33}$ Chondrocytes are the only cell type in the articular cartilage, responsible for the homeostasis of ECM through the synthesis of collagen II and aggrecan. The degradation of articular cartilage is caused by the principal matrix degrading enzymes. Two main categories of enzymes participate in 


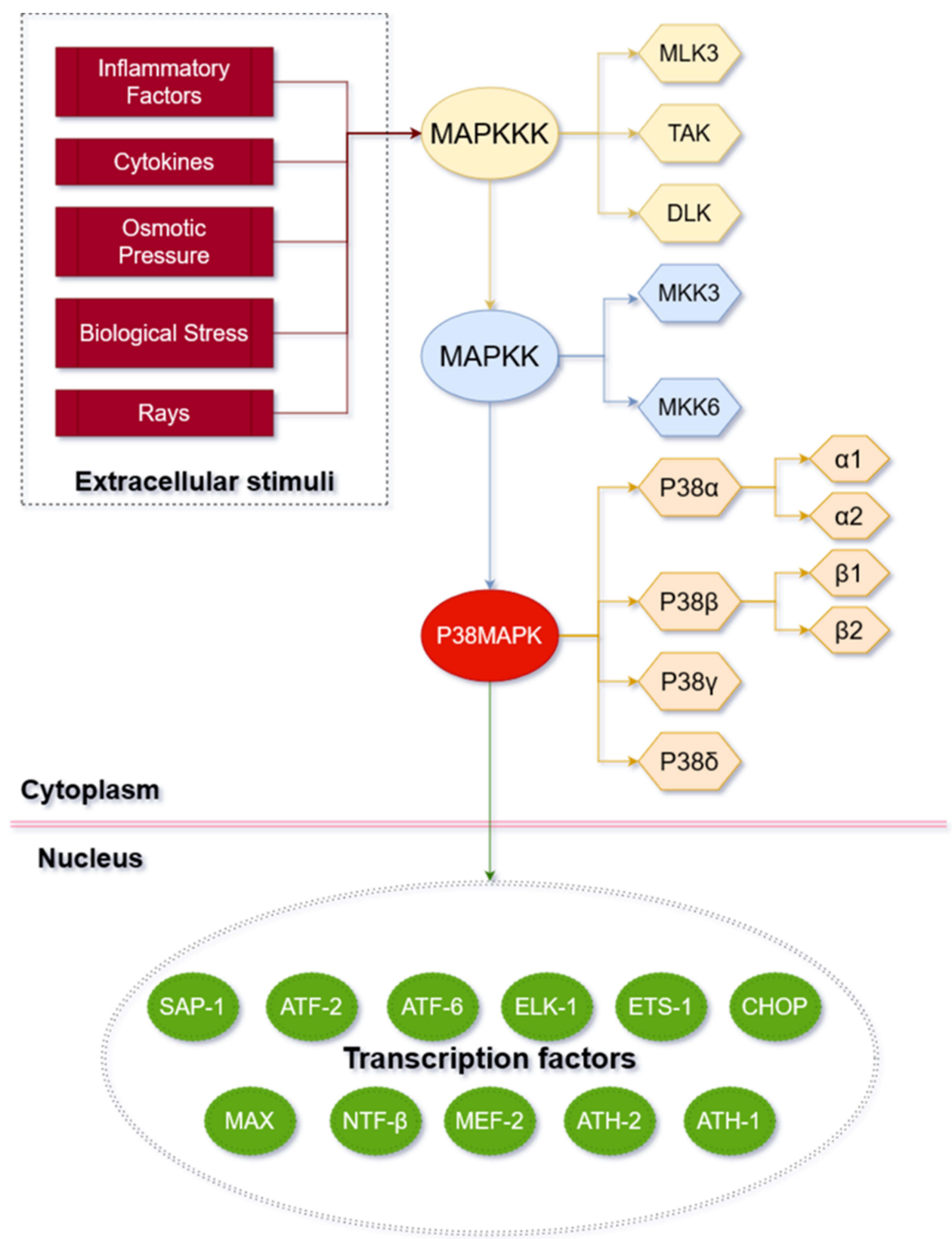

Figure I Basic mechanism of p38MAPK pathway. The P38MAPK signaling pathway involves three key kinases: MAPKKK (beige box), MAPKK (blue box), and MAPK (orange box). P38MAPK signaling pathway can be affected by extracellular stimuli (red box) and then enter the nucleus to active transcription factors (green box).

this process: the disintegrin-like and metalloproteinase with the thrombospondin motifs (ADAMTS) family and the MMPs, which degrade aggrecan (ADAMTS) and collagen (MMPs) respectively. ${ }^{34}$

The interconnection between the p38MAPK signaling pathway and ADAMTs has not yet been elucidated clearly. However, sufficient evidence has supported that the p38MAPK signaling pathway can progressively suppress the synthesis and increase the degradation of type II collagen by inducing the expression of MMPs, thus accelerating cartilage destruction and the course of OA ${ }^{35-37}$ MMPs are a family of proteinases that is responsible for regulating ECM turnover. Both MMP-1 and MMP-13 have significant effects on the degradation of the OA cartilage matrix. The difference lies in that MMP-13 is more inclined to lyse type II over type I and III collagen, while MMP-1 is more inclined to lyse type III and MMP-8 type I collagen. ${ }^{38}$ Yu et al found that Interferon $\alpha 2 \mathrm{~b}$ exerted an anti-fibrosis effect in a MAPK signaling pathway-dependent manner by increasing the level of MMP-13 and IL-10 in Kupffer cells. ${ }^{39}$ 


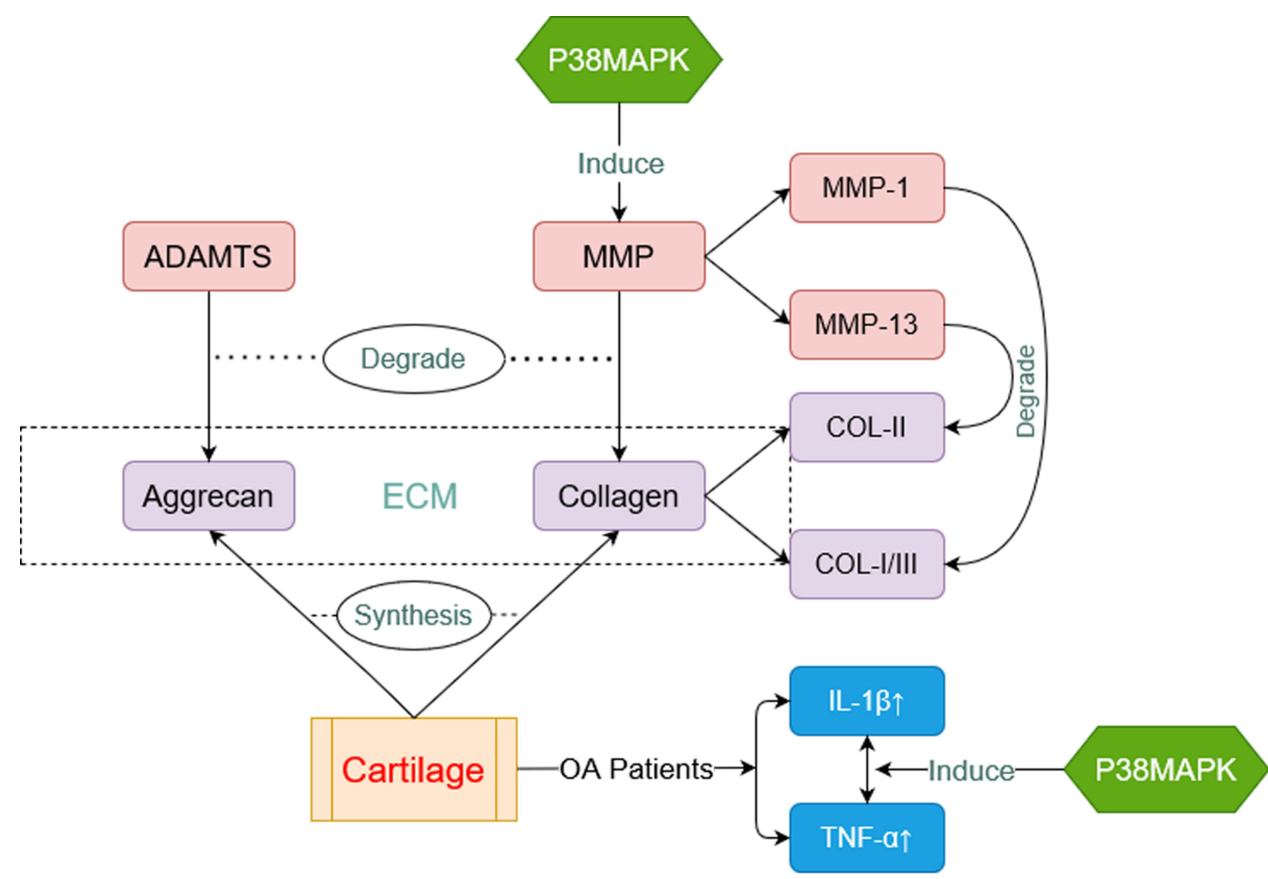

Figure 2 Pathophysiology of OA related to p38MAPK. Aggrecan and collagen (purple box) are major components of cartilage. They are able to decomposed by a disintegrin-like and metalloproteinase with the thrombospondin motifs (ADAMTS) and matrix metalloproteinase (MMP) (red box) respectively. P38MAPK can induce expressions of MMP. IL- $\beta$ and TNF- $\alpha$ (blue box) are always at a high level in cartilage of OA Patients. P38MAPK is also involved in this process.

\section{IL-I $\beta$ and TNF- $\alpha$}

Another key pathophysiological change in $\mathrm{OA}$ is the presence of inflammatory factors. The role of catabolic or proinflammatory cytokines, such as IL-1 and TNF- $\alpha$, has been demonstrated in the pathophysiology of OA. ${ }^{40}$ Cytokine inhibitors have already been applied as a treatment of OA, which has received promising results. ${ }^{41}$ Common inflammatory factors include IL-1 $\beta$, IL-6, IL-15, IL-17, IL-18, as well as the tumor necrosis factor (TNF- $\alpha$ ); in particular, IL-1 $\beta$ and TNF- $\alpha$ have been well studied. ${ }^{42}$ IL-1 is the most classic inflammatory regulator and the initiating factor of inflammation. As early as in the 1970s, Howell et al discovered that the supernatant of macrophages cultured in vitro could promote the degradation of cartilage and promote the secretion of metalloproteinases by chondrocytes. IL- $1 \beta$, a subtype of IL-1, is produced primarily by activated mononuclear macrophages. Studies have shown that the serum level of IL- $1 \beta$ is related to OA progression, and elevated levels of IL-1 $\beta$ are found in the synovial fluid, synovial membrane, cartilage, and subchondral tissue in patients with OA. ${ }^{43} \mathrm{IL}-1 \beta$ has been reported to be involved in a variety of signaling pathway mediated OA processes, such as P38MAPK, Wnt, and NF-kB. ${ }^{15,44}$ As one of the IL-1 inhibitors, Diacerein has been widely used in clinical practice. ${ }^{45}$ There is another inhibitor, lutikizumab, which is being intensively studied recently. ${ }^{46}$ However, it did not significantly alleviate OA patients' pain in Phase II clinical trials, preventing it to be a new hope to cure OA. ${ }^{46}$

TNF- $\alpha$ is mainly produced by macrophages, fibroblasts and chondrocytes. Elevated levels of TNF- $\alpha$ have been detected in all sites, accompanied with elevated levels of IL- $1 \beta .{ }^{47} \mathrm{TNF}-\alpha$ can bind to two membrane receptors, TNF-R1 and TNF-R2. Specifically, TNF-R1 can be activated effectively in the dissolved state and in the membrane form, while TNF-R2 can only be activated in the membrane form. ${ }^{48}$ At present, TNF-R1 has been shown to have a greater effect on cartilage destruction than TNF-R2, but they both play an important role in signal transduction. The binding of TNF- $\alpha$ and TNF-R1 leads to the interaction of TNFR-associated death domain protein (TRADD) binding proteins with other binding egg whites (eg, TRAF2, CIAP1, CIAP2, and RIP1). The produced complex is then ubiquitinated by RIP1 and binds to TAK1, Tables 1 and 2, eventually leading to phosphorylation of IKK and activation of the transcription factor NF- $\mathrm{KB} .{ }^{40}$ The similarity between TNF- $\alpha$ and IL- $1 \beta$ is that the MAPK signaling pathway is activated in all of these physiological processes. The activated pathway transmits intracellular signals rapidly, which will lead to altered expressions of related 
Table I Factors Affecting P38MAPK and Progression of OA

\begin{tabular}{|c|c|c|c|c|}
\hline Authors (Year) & Samples & Factors & Pathway(via) & Findings \\
\hline Liao et al $(2020)^{12}$ & 30C57bl/6 Mice & $\begin{array}{l}\text { Advanced oxidation } \\
\text { protein products } \\
\text { (AOPPs) }\end{array}$ & $\begin{array}{l}\text { P38MAPK, } \\
\text { NADPH oxidase4- } \\
\text { dependent, }\end{array}$ & $\uparrow T N F-\alpha$, IL-I $\beta$ \\
\hline Shan et al $(2019)^{13}$ & Normal Human Cartilage & $\begin{array}{l}\text { Angiopoietin-like } 2 \\
\text { (ANGPTL2) }\end{array}$ & $\begin{array}{l}\text { P38MAPK, NF-кB } \\
\text { (integrin } \alpha 5 \beta \mathrm{I} \text { ) }\end{array}$ & $\begin{array}{l}\uparrow I L-I \beta, \text { IL-6 } \\
\downarrow E C M\end{array}$ \\
\hline Ma et al $(2019)^{14}$ & $\begin{array}{l}\text { Human Bone Marrow } \\
\text { Mesenchymal Stem Cells (BMSCs) }\end{array}$ & TGF- $\beta I$ & $\begin{array}{l}\text { P38MAPK, ERK, } \\
\text { JNK, Smad }\end{array}$ & $\uparrow$ SOX9, Collagen II, Aggrecan, GAG \\
\hline Han et al $(2020)^{50}$ & OA patients' Knee Cartilage & $\begin{array}{l}\text { Kruppel like factors II } \\
\text { (KLFII) }\end{array}$ & P38MAPK & $\begin{array}{l}\downarrow \text { oxidative stress levels, apoptosis } \\
\text { levels, activity of ERS-related } \\
\text { pathways }\end{array}$ \\
\hline $\begin{array}{l}\text { Wang et al } \\
(2017)^{91}\end{array}$ & $\begin{array}{l}\text { Dawley rats, weighing } 300-400 \\
g \text { ( } 5 \text { females and } 5 \text { males })\end{array}$ & $\begin{array}{l}\text { Pyruvate dehydrogenase } \\
\text { kinase isoform } 2 \text { (PDK2) }\end{array}$ & $\begin{array}{l}\text { P38MAPK, ERK, } \\
\text { JNK }\end{array}$ & $\uparrow$ mesenchymal stem cells (MSCs) \\
\hline Lei et al $(2019)^{51}$ & Normal Human Cartilage & $\begin{array}{l}\text { LncRNA small nucleolar } \\
\text { RNA host gene I } \\
\text { (SNHGI) }\end{array}$ & $\begin{array}{l}\text { p38MAPK, NF-кB } \\
(\mathrm{miR}-16-5 \mathrm{p})\end{array}$ & $\begin{array}{l}\downarrow N O, \text { PGE2, IL-6, TNF- } \alpha \text {, i-NOS, } \\
\text { COX-2, MMPs, ADAMTs }\end{array}$ \\
\hline Kang et al $(2016)^{58}$ & $\begin{array}{l}\text { Young (2-week-old) and old } \\
\text { (2-year-old) New Zealand White } \\
\text { rabbits }\end{array}$ & $\begin{array}{l}\text { Cyclosporine A (CsA) } \\
\text { and tacrolimus (FK506) }\end{array}$ & P38MAPK & $\begin{array}{l}\downarrow \text { Senescence of articular } \\
\text { chondrocytes }\end{array}$ \\
\hline $\begin{array}{l}\text { Benabdoune et al } \\
(2016)^{92}\end{array}$ & OA patients' Knee Cartilage & Resolvin DI & $\begin{array}{l}\text { P38MAPK, JNK, } \\
\text { NF-kB (IL-I } \beta)\end{array}$ & $\begin{array}{l}\uparrow B c \mid 2, A K T, \text { GSH. } \\
\downarrow \text { lactate dehydrogenase release }\end{array}$ \\
\hline $\begin{array}{l}\text { Zheng et al } \\
(2020)^{93}\end{array}$ & Adult SD rats & CTRP9 & P38MAPK, NF-кB & $\begin{array}{l}\uparrow I L-I \beta, \text { IL-6 } \\
\downarrow M M P s\end{array}$ \\
\hline
\end{tabular}

Table 2 The Natural Products and Traditional Chinese Medicine Extracts of p38MAPK

\begin{tabular}{|c|c|c|c|c|}
\hline Authors (Years) & Samples & Extracts & Regulate & Pathways \\
\hline Ren et al $(2018)^{79}$ & ATDC5 cells & Piperine & miR-127, MyD88 & NF- $\kappa B, ~ р 38 M A P K$ \\
\hline Fan et al $(2019)^{80}$ & ATDC5 cells & Astragalus polysaccharide (APS) & miR-92a, KLF4 axis & NF- $\kappa B, p 38 M A P K$ \\
\hline Jin et al $(2018)^{81}$ & ATDC5 cells & Resveratrol (RSV) & miR-146b & NF- $\kappa B, p 38 M A P K$ \\
\hline $\mathrm{Li}$ et al $(2019)^{82}$ & ATDC5 cells & Tetramethylpyrazine (TMP) & MyD88 & NF- $\kappa B, ~ p 38 M A P K$ \\
\hline Feng et al $(2020)^{83}$ & OA patients' Cartilage & Salvianolic acid A (SAA) & $\begin{array}{l}\uparrow \text { Collagen II, Aggrecan. } \\
\downarrow M M P I, \text { MMPI } 3 \text {, } \\
\text { ADAMTS-5 }\end{array}$ & NF-kB, p38MAPK \\
\hline Li et al $(202 \mathrm{I})^{84}$ & KOA model mice & Wang-Bi Tablet (WBT) & $\begin{array}{l}\uparrow \text { glycosaminoglycans } \\
\downarrow \text { IL-6 }\end{array}$ & NF-кB, p38MAPK \\
\hline
\end{tabular}

genes and increased secretion of inflammatory factors, chemokines, adhesion factors, inflammatory mediators and enzymes. ${ }^{49}$ Whether inflammatory factors are the cause of or have any effect on cartilage destruction remains unclear. However, it has been confirmed that the p38MAPK signaling pathway is an important regulator. A various of inhibitors or non-coding RNAs(ncRNAs) inhibit the p38MAPK signaling pathway and down-regulate IL-1 $\beta$ and TNF- $\alpha$ expression, thereby reducing the progression of $\mathrm{OA} .^{50-52}$

\section{Regulatory Roles and Potential Targets of p38MAPK Pathway in OA}

It has been well established that p38MAPK is involved in multiple systemic chronic diseases such as OA, Alzheimer's disease, heart disease, chronic obstructive pulmonary disease, renal fibrosis and obesity. ${ }^{53-57}$ Most of these diseases are associated with chronic systemic inflammation, and the course of disease is difficult to be completely reversed. ${ }^{54-57}$ Pathologically, OA is featured by the degeneration of cartilage. The mechanism that initiates chondrocyte changes and 
cartilage damage in OA is not clear. At present, it is believed that the p38MAPK signaling pathway plays a pivotal role in the cartilage degeneration process in OA, and the degeneration of cartilage and damage of chondrocytes will further promote the process of OA. ${ }^{50,52,58}$ Zhang et al created articular cartilage defect rabbit knee joint models to detect the influence of Notch inhibitor (DAPT) combined with p38MAPK inhibitor (SB203580) on cartilage degradation. ${ }^{59}$ Compared with the control group, the inhibition of the Notch/p38MAPK signaling pathway was found to promote the recovery of articular cartilage, accelerate chondrocyte proliferation, and promote the recovery of knee injury in rabbits.

In recent years, an increasing number of upstream activators or inhibitors of the MAPK pathway that can regulate the function of p38MAPK signaling pathway and affect the progression of OA have been discovered. Liao et al established a rodent OA model via anterior cruciate ligament transection (ACLT) and found that advanced oxidation protein products (AOPPs) were deposited in the model cartilage. ${ }^{12}$ The authors' further investigation confirmed that AOPPs increased the expressions of TNF- $\alpha$ and IL- $1 \beta$ in mice's chondrocytes through the p38MAPK pathway and accelerated the cartilage degeneration in the progression of OA. Moreover, apocynin (NADPH oxidase inhibitor) decreased the expressions of TNF- $\alpha$ and IL-1 $\beta$ and attenuated the progression of OA (the OARSI scores were decreased in the group added with apocynin). According to the study by Shan et al, the upregulation of angiopoietin-like 2 (ANGPTL2) exacerbated the chondrocyte injury through the NF- $\mathrm{KB}$ and p38MAPK signaling pathways. ${ }^{13}$ In the human OA cartilage, ANGPTL2 is upregulated, which consequently activates NF-KB and p38/MAPK signaling via integrin $\alpha 5 \beta 1$ in primary chondrocytes. In addition, Zhou et al reported that the OA infrapatellar fat pad (IPFP) induced cartilage degradation and inflammation by activating the p38MAPK and ERK1/2 pathways, in which IL- $1 \beta$ and TNF- $\alpha$ are key factors. They further proposed that modulating the effect of IPFP on cartilage might be a promising intervention strategy for knee OA. ${ }^{60}$

Recently, the interaction between p38MAPK and pro-inflammatory factors has drawn growing attention, and a considerable number of new studies have been conducted. The activated transcription factor (ATF) 2 and myocyte enhancing-factor (MEF) 2C are the main substrates of p38MAPK. ${ }^{61,62}$ The phosphorylation of ATF2 regulates the production of a variety of inflammatory cytokines, including but not limited to TNF- $\alpha$, IL-1, IL- 6 , etc., and the phosphorylation of $\mathrm{MEF} 2 \mathrm{C}$ upregulates the transcription of the transcription factor c-Jun, thereby elevating its expression. Phosphorylated c-Jun under the inflammatory state can bind to the activator protein (AP)-1 sites in many cytokine promoter regions, which will subsequently increase the expressions of inflammation-related genes, thereby playing an important role in the inflammatory process. ${ }^{63}$ As reported by Ma et al, ${ }^{14}$ activation of the p38MAPK pathway promoted the chondrogenic differentiation induced by TGF- $\beta 1$, while inhibition of the p38 pathway could inhibit chondrogenic differentiation. This finding may have a guiding value to the treatment of OA. Sun et al ${ }^{15}$ argued that inhibition of the p38MAPK signaling pathway could suppress the apoptosis of human OA chondrocytes and the production of proinflammatory cytokines. In the group treated with the p38 pathway inhibitor SB203580, lower serum levels of IL-1, IL-6, and TNF-a were observed compared with the control group. The p38MAPK signaling pathway is closely related to inflammatory factor production, which explains its effect on the progression of OA. The specific research contents are summarized in Table 1.

\section{NcRNAs in the Regulation of p38MAPK Function}

NcRNAs are a class of RNA molecules that do not encode for proteins but function to modulate cell physiology, regulate cellular processes and shape cellular functions. ncRNAs account for more than $90 \%$ of the total RNA in cells, among which microRNAs (miRNAs) are important members. miRNAs is an endogenous and genetically, highly conserved single-stranded non-programmed small RNA with a length of 19-25 bases. miRNAs have been demonstrated to play important roles in immunity, inflammation, infection and metabolism by binding to 3'-UTR, so as to regulate the expressions of related genes. ${ }^{64} \mathrm{~A}$ large number of studies in the past decades have revealed that the occurrence and development of OA is related to pro-inflammatory factors, cell apoptosis, MMPs secretion and the hydrolyzed protein of VHF. ${ }^{65}$ Studies have also shown that miRNAs may become a new indicator for OA diagnosis and drug development.

Among the miRNAs associated with OA, some are closely linked with the p38MAPK signaling pathway. Lei et al suggested that the long non-coding RNA, small nucleolar RNA host gene 1 (SNHG1), lowered the expressions of MMPs, ADAMTs, collagen, and pro-inflammatory cytokines, and could directly interact with miR-16-5p to suppress the miR-16- 
$5 \mathrm{p}$ expression. ${ }^{51}$ Furthermore, SNHG1 was indicated to alleviate the IL-1 $\beta$-induced OA inflammation by activating the miR-16-5p mediated p38MAPK and NF- $\kappa$ B signaling pathways. ${ }^{51}$ Zarfa et al examined the effect of hsa-miR-125b-5p on the IL-1 $\beta$-induced inflammatory genes in human OA chondrocytes. The authors reported that hsa-miR-125b-5p played a negative synergistic role in the regulation of inflammatory genes, including MMP-13, by targeting the TRAF6/MAPKs/ NF- $\kappa B$ pathway in human OA chondrocytes. ${ }^{66}$ Yuan et al and Cao et al revealed a close relationship between miR-124, miR-296-5p and p38MAPK, respectively. ${ }^{52,67}$ From what has been reported in the existing literature, miRNAs has been shown to regulate the p38MAPK targeted genes, and consequently, affect the cartilage and chondrocytes through the p38MAPK signal pathway, thereby interfering with the progression of OA.

\section{p38MAPK Pathway as Therapeutic Targets in OA Treatment}

The activation of the p38MAPK signaling pathway is involved in the pathological process of OA, making it a potential treatment for OA by inhibiting this pathway. Current available approaches to inhibit the p38MAPK signaling pathway can be divided into two types: biochemical inhibitors and natural products.

The research of small molecule p $38 \alpha$ inhibitors developed rapidly in the last decade and hundreds of reports have been published. Specifically, the small molecule P38 $\alpha$ inhibitors that are currently under clinical study can be roughly divided into pyridine imidazoles, pyridines, pyridopyrimidines, indoles, ureas and others. Among these inhibitors, SB203580 (belonging to the class of imidazopyridines compounds) is the most widely used one. SB203580 is also the first class of selective p38 inhibitors to be synthesized artificially. ${ }^{68}$ Instead of blocking the activation of $\mathrm{p} 38$ by affecting the upstream kinase, it binds to ThrL06, the ATP-binding active site of p38. As a result, p38 loses its ability to bind to ATP and will eventually lose its kinase activity. ${ }^{69}$ However, SB203580 is mostly used in vitro or in animal experiments due to low drug selectivity and a high rate of toxic side effects. ${ }^{70-72}$ Other types of inhibitors, including pyridines, pyrimidines and indoles, also have the same problem. Pamapimod (PAM), a novel p38MAPK inhibitor firstly discovered in 2008, could improve the signs and symptoms of RA and other autoimmune diseases. ${ }^{73}$ In recent studies, Zhao et al reported that PAM could prevent ovariectomized-induced bone loss by inhibiting p38-induced osteoclast formation and bone resorption; ${ }^{74}$ Zhang et al found that PAM protected chondrocytes from hypertrophy by inhibiting the p38 pathway; ${ }^{75}$ and Zhao et al examined the emerging role of Resistin on the pathogenesis of OA. ${ }^{76}$ In the human cartilage, Resistin induces the expressions of proinflammatory factors, such as degradative enzymes, to inhibit cartilage matrix synthesis. This is achieved perhaps by binding to the Toll-like receptor 4 and the adenylyl cyclase-associated protein 1 receptor, which then activates the p38MAPK. ${ }^{76}$ A similar point of view was also claimed by Chen et al. ${ }^{77}$ They found that Resistin enhanced the expression of vascular cell adhesion molecule type 1 and monocyte adhesion in human OA synovial fibroblasts by inhibiting miR-381 expression through the p38 signaling pathway. Thus, PAM and Resistin may be regarded as novel therapeutic targets for OA treatment in the future. In addition, Huang et al reported that the protease activated receptor 2 (PAR2) antagonist AZ3451 attenuated the apoptosis of chondrocytes by activating autophagy in vitro, ${ }^{78}$ while in vivo, the intraarticular injection of AZ3451 ameliorated cartilage degeneration in surgically induced rat OA models. Furthermore, the p38MAPK, NF- $\mathrm{kB}$ and PI3K/ Akt /mTOR pathways are involved in the protective mechanism of AZ3451. ${ }^{78}$ Therefore, AZ3451 may serve as a promising strategy for OA treatment.

In addition to synthetic substances, an increasing number of natural products and extracts of Traditional Chinese medicine (TCM) have also been proven to inhibit OA to varying degrees. ${ }^{79-84}$ Due to the complex composition of TCM, there was no sufficient evidence supporting its role in delaying the process of OA in the past. However, the effects of TCM exacts have been fully demonstrated in relevant experiments that were carried out recently. The ATDC5 cell line is derived from the AT805 mouse teratoid carcinoma line. As a prechondral cell line, its differentiation process is similar to that of chondrogenesis. Therefore, it is often used in cartilage related experimental studies. ${ }^{85}$ Most of the experiments on TCM extracts induced ATDC5 cell damage using LPS so as to simulate the process of $\mathrm{OA} .^{79-82}$ It has been revealed that TCM extracts could regulate miRNAs to inhibit P38MAPK, NF- $\mathrm{B}$ and other signaling pathways, thereby delaying the progression of $\mathrm{OA}^{79-82}$ An experiment using cells from patients with OA proved that Salvianolic acid A (SAA) inhibited the expressions of MMP-1, MMP13 and ADAMTS-5, and improved the synthesis of type II collagen and aggregates by down-regulating NF- $\mathrm{B}$ and 
Table 3 The Other Inhibitors of p38MAPK

\begin{tabular}{|l|l|l|l|}
\hline Authors (Years) & Names & Research Field & Subject \\
\hline Noguchi et al $(2020)^{86}$ & IIR-p38I 110 & Islet & Porcine \\
Damjanov et al $(2009)^{88}$ & VX-702 & RA & RA patients \\
Wagner et al $(2015)^{87}$ & VX-702 & Platelet & Platelet \\
Ito et al (2008) & P38IH & Pancreas & Canine \\
Bagley et al (20I6) & RO320II95 & Werner syndrome & Werner syndrome cells \\
\hline
\end{tabular}

p38MAPK pathways. ${ }^{83}$ Another experiment based on knee OA (KOA) mice model showed that Wang-Bi tablets (WBTs) could alleviate inflammation and prevent cartilage injury also by down-regulating NF- $\mathrm{kB}$ and p38MAPK pathways. ${ }^{84}$ However, no human experiment at present can support these arguments, so there is still a lack of higher-level evidence. The detailed contents of the literature above are presented in Table 2.

\section{Future Research}

To date, no method is available to reverse the progression of OA. Pharmacological treatments for OA are limited to symptomatic relief but not disease modification. By targeting p38MAPK, the inhibitors like SB203580 and PAM may become a new hope for the treatment of OA. However, further investigation is needed to figure out how SB203580, as a competitive inhibitor of ATP, selectively inhibits the target without affecting the interaction between other enzymes in vivo. PAM was once considered as a potential drug for rheumatoid arthritis (RA) treatment. Research on its association with OA is still insufficient, but it provides referential significance as whether the inhibitors of p38 applied in other diseases can be used to treat OA. Noguchi et al designed the Peptide $11 \mathrm{R}-\mathrm{p} 38 \mathrm{I}_{110}$, a novel cellpermeable p38MAPK inhibitor, and found that it could prevent porcine islet apoptosis and improve islet graft function. ${ }^{86}$ VX-702, a novel p38MAPK inhibitor, has been used in the research of RA and platelet. ${ }^{87,88}$ The details about the various inhibitors of p38 are listed in Table 3. TCM extracts are also a promising innovative approach, which requires support from more in-depth research and further long-term clinical trials. ${ }^{79-83}$ In addition, there are many diseases being regulated by the p38MAPK signaling pathway, and most of these diseases involve chronic systemic inflammation and are closely related to aging. ${ }^{53-57}$ For example, MCC950, the NLRP3 Inhibitor, has antiinflammatory and anti-senescence effects on some chronic diseases such as Alzheimer's disease. ${ }^{89}$ In a recent study, $\mathrm{Ni}$ et al found that the NLRP3 protein was upregulated in the human and mouse OA cartilage and the intra-articular administration of MCC950 could postpone cartilage degeneration. ${ }^{90}$ Therefore, MCC950 might play a protective role in the cartilage by regulating p38MAPK. ${ }^{90}$ The interconnection between p38MAPK regulated inflammation and aging, as well as the potential synergistic/antagonistic effects between the inhibitors of different diseases, are worth exploring and studying.

\section{Conclusions}

The pathophysiology of OA related to p38MAPK has not been sufficiently studied. A variety of in vitro and in vivo experiments have shown that inhibiting the p38MAPK signaling pathway can suppress chondrocyte apoptosis, promote cartilage repair, inhibit the production of inflammatory factors, and delay the course of OA. More research on the p38MAPK signaling pathway is needed to better understand its regulatory role in OA and potential for translational studies. The p38MAPK inhibitors may become a new therapy of OA.

\section{Funding}

This study was supported by the National Natural Science Foundation of China (81902303), the Natural Science Foundation of Hunan Province (No.2020JJ8043), Guangdong Basic and Applied Basic Research Foundation (2020A151501048), the Key project of Hunan Provincial Health Commission (No.20201902), the Project of Hunan Provincial Health Commission (No. c2019133), Shenzhen Science and Technology Project (JCYJ20190806164216661, RCBS20200714114856299), the Science and Technology Project of Changsha City (No. kq2004165), the Hunan 
Provincial Clinical Medical Technology Innovation Guiding Project (No. 2020SK53307), and Clinical Research Project of Shenzhen Second People's Hospital (20203357028).

\section{Disclosure}

The authors report no conflicts of interest in this work.

\section{References}

1. Hunter DJ, Bierma-Zeinstra S. Osteoarthritis. Lancet. 2019;393(10182):1745-1759. doi:10.1016/S0140-6736(19)30417-9

2. Glyn-Jones S, Palmer AJR, Agricola R, et al. Osteoarthritis. Lancet. 2015;386(9991):376-387. doi:10.1016/S0140-6736(14)60802-3

3. James Spencer L, Abate D, Abate Kalkidan H, et al. Global, regional, and national incidence, prevalence, and years lived with disability for 354 diseases and injuries for 195 countries and territories, 1990-2017: a systematic analysis for the Global Burden of Disease Study 2017. Lancet. 2018;392(10159):1789-1858.

4. Sellam J, Berenbaum F. The role of synovitis in pathophysiology and clinical symptoms of osteoarthritis. Nat Rev Rheumatol. 2010;6(11):625-635. doi:10.1038/nrrheum.2010.159

5. Palazzo C, Nguyen C, Lefevre-Colau M, Rannou F, Poiraudeau S. Risk factors and burden of osteoarthritis. Ann Phys Rehabil Med. 2016;59 (3):134-138. doi:10.1016/j.rehab.2016.01.006

6. Le Graverand-gastineau MH. Disease modifying osteoarthritis drugs: facing development challenges and choosing molecular targets. Curr Drug Targets. 2010;11(5):528. doi:10.2174/138945010791011893

7. Zhang W, Ouyang H, Dass CR, Xu J. Current research on pharmacologic and regenerative therapies for osteoarthritis. Bone Res. 2016;4(1):15040. doi:10.1038/boneres.2015.40

8. Santana DC, Emara AK, Orr MN, et al. An update on venous thromboembolism rates and prophylaxis in hip and knee arthroplasty in 2020. Medicina. 2020;56(9):416. doi:10.3390/medicina56090416

9. SooHoo NF. Factors predicting complication rates following total knee replacement. J Bone Joint Surg. 2006;88(3):480.

10. Shi J, Zhang C, Yi Z, Lan C. Explore the variation of MMP3, JNK, p38 MAPKs, and autophagy at the early stage of osteoarthritis. Iubmb Life. 2016;68(4):293-302. doi:10.1002/iub.1482

11. Gao T, Hu Q, Hu X, et al. Novel selective TOPK inhibitor SKLB-C05 inhibits colorectal carcinoma growth and metastasis. Cancer Lett. 2019;445:11-23. doi:10.1016/j.canlet.2018.12.016

12. Liao $\mathrm{C}$, Wang $\mathrm{S}$, Zhu S, et al. Advanced oxidation protein products increase TNF- $\alpha$ and IL-1 $\beta$ expression in chondrocytes via NADPH oxidase 4 and accelerate cartilage degeneration in osteoarthritis progression. Redox Biol. 2020;28:101306. doi:10.1016/j.redox.2019.101306

13. Shan W, Cheng C, Huang W, et al. Angiopoietin-like 2 upregulation promotes human chondrocyte injury via NF- $\mathrm{kB}$ and $\mathrm{p} 38 / \mathrm{MAPK}$ signaling pathway. J Bone Miner Metab. 2019;37(6):976-986. doi:10.1007/s00774-019-01016-w

14. Ma N, Teng X, Zheng Q, Chen P. The regulatory mechanism of p38/MAPK in the chondrogenic differentiation from bone marrow mesenchymal stem cells. J Orthop Surg Res. 2019;14(1):434. doi:10.1186/s13018-019-1505-2

15. Sun H, Hu K, Yin Z. Inhibition of the p38-MAPK signaling pathway suppresses the apoptosis and expression of proinflammatory cytokines in human osteoarthritis chondrocytes. Cytokine. 2017;90:135-143. doi:10.1016/j.cyto.2016.11.002

16. Brewster JL, de Valoir T, Dwyer ND, Winter E, Gustin MC. An osmosensing signal transduction pathway in yeast. Science. 1993;259 (5102):1760-1763. doi:10.1126/science.7681220

17. Han J, Lee JD, Bibbs L, Ulevitch RJ. A MAP kinase targeted by endotoxin and hyperosmolarity in mammalian cells. Science. 1994;265 (5173):808-811. doi:10.1126/science.7914033

18. Johnson GL, Lapadat R. Mitogen-activated protein kinase pathways mediated by ERK, JNK, and p38 protein kinases. Science. 2002;298 (5600):1911-1912. doi:10.1126/science.1072682

19. Morazzani M, de Carvalho DD, Kovacic H, et al. Monolayer versus aggregate balance in survival process for EGF-induced apoptosis in A431 carcinoma cells: implication of ROS-P38 MAPK-integrin ?2?1 pathway. Int J Cancer. 2004;110(6):788-799. doi:10.1002/ijc.20198

20. Wang XS, Diener K, Manthey CL, et al. Molecular cloning and characterization of a novel p38 mitogen-activated protein kinase. J Biol Chem. 1997;272(38):23668-23674. doi:10.1074/jbc.272.38.23668

21. Liu J, Han L, Li B, et al. F-box only protein 31 (FBXO31) negatively regulates p38 mitogen-activated protein kinase (MAPK) signaling by mediating lysine 48-linked ubiquitination and degradation of mitogen-activated protein kinase kinase 6 (MKK6). J Biol Chem. 2014;289 (31):21508-21518. doi:10.1074/jbc.M114.560342

22. Sanderson TM, Hogg EL, Collingridge GL, Corrêa SAL. Hippocampal metabotropic glutamate receptor long-term depression in health and disease: focus on mitogen-activated protein kinase pathways. J Neurochem. 2016;139:200-214. doi:10.1111/jnc.13592

23. Kojonazarov B, Novoyatleva T, Boehm M, et al. p38 MAPK inhibition improves heart function in pressure-loaded right ventricular hypertrophy. Am J Resp Cell Mol. 2017;57(5):603-614. doi:10.1165/rcmb.2016-0374OC

24. Xu Q, Fang H, Zhao L, et al. Mechano growth factor attenuates mechanical overload-induced nucleus pulposus cell apoptosis through inhibiting the p38 MAPK pathway. Bioscience Rep. 2019;39(3). doi:10.1042/BSR20182462

25. Kita K, Sugita K, Sato C, et al. Extracellular release of annexin A2 is enhanced upon oxidative stress response via the p38 MAPK pathway after low-dose X-ray irradiation. Radiat Res. 2016;186(1):79. doi:10.1667/RR14277.1

26. Lee JC, Kumar S, Griswold DE, et al. Inhibition of p38 MAP kinase as a therapeutic strategy. Immunopharmacology. 2000;47(2-3):185-201. doi:10.1016/S0162-3109(00)00206-X

27. Martín-Segura A, Casadomé-Perales Á, Fazzari P, et al. Aging increases hippocampal DUSP2 by a membrane cholesterol loss-mediated RTK/ p38MAPK activation mechanism. Front Neurol. 2019;10:675. doi:10.3389/fneur.2019.00675

28. Brichkina A, Bulavin DV. Cancer suppression by systemic inactivation of p38MAPK. Oncotarget. 2017;8(9):14275-14276. doi:10.18632/ oncotarget.15293 
29. Moreno-Cugnon L, Arrizabalaga O, Llarena I, Matheu A. Elevated p38MAPK activity promotes neural stem cell aging. Aging (Albany NY). 2020;12(7):6030-6036. doi:10.18632/aging.102994

30. Huang J, Liu C, Ming X, Yang Z. Inhibition of p38mapk reduces adipose tissue inflammation in aging mediated by arginase-II. Pharmacology. 2020;105(9-10):491-504. doi:10.1159/000507635

31. Chen X, Zhang P, Ma X. Rab7 delays intervertebral disc degeneration through the inhibition of the p38MAPK pathway. Biochem Bioph Res Commun. 2019;514(3):835-841. doi:10.1016/j.bbrc.2019.04.184

32. Kheiri G, Dolatshahi M, Rahmani F, Rezaei N. Role of p38/MAPKs in Alzheimer's disease: implications for amyloid beta toxicity targeted therapy. Rev Neurosci. 2018;30(1):9-30. doi:10.1515/revneuro-2018-0008

33. Sherwood J. Osteoarthritis year in review 2018: biology. Osteoarthr Cartilage. 2019;27(3):365-370. doi:10.1016/j.joca.2018.10.005

34. Cui N, Hu M, Khalil RA. Biochemical and biological attributes of matrix metalloproteinases. Prog Mol Biol Transl. 2017;147:1-73.

35. Mehana EE, Khafaga AF, El-Blehi SS. The role of matrix metalloproteinases in osteoarthritis pathogenesis: an updated review. Life Sci. 2019;234:116786. doi:10.1016/j.lfs.2019.116786

36. Slovacek H, Khanna R, Poredos P, et al. Interrelationship of osteopontin, MMP-9 and ADAMTS4 in patients with osteoarthritis undergoing total joint arthroplasty. Clin Appl Thrombos Hemostas. 2020;26:107602962096486. doi:10.1177/1076029620964864

37. Niu C, Lin S, Yuan L, et al. Upregulation of miR-107 expression following hyperbaric oxygen treatment suppresses HMGB1/RAGE signaling in degenerated human nucleus pulposus cells. Arthritis Res Ther. 2019;21(1):42. doi:10.1186/s13075-019-1830-1

38. Nagase H, Visse R, Murphy G. Structure and function of matrix metalloproteinases and TIMPs. Cardiovasc Res. 2006;69(3):562-573. doi:10.1016/ j.cardiores.2005.12.002

39. Yu Z, Xie M, Fan X, Jia J. Interferon alpha2b increases MMP-13 and IL-10 expression in Kupffer cells through MAPK signaling pathways. Hepatogastroenterology. 2015;62(138):350-354.

40. Kapoor M, Martel-Pelletier J, Lajeunesse D, Pelletier J, Fahmi H. Role of proinflammatory cytokines in the pathophysiology of osteoarthritis. Nat Rev Rheumatol. 2011;7(1):33-42. doi:10.1038/nrrheum.2010.196

41. McAlindon TE, Bannuru RR, Sullivan MC, et al. OARSI guidelines for the non-surgical management of knee osteoarthritis. Osteoarthr Cartilage. 2014;22(3):363-388. doi:10.1016/j.joca.2014.01.003

42. Kim J, Yoo J, Kim H. Therapeutics in osteoarthritis based on an understanding of its molecular pathogenesis. Int J Mol Sci. 2018;19(3):674. doi:10.3390/ijms19030674

43. Vincent TL. IL-1 in osteoarthritis: time for a critical review of the literature. F1000Research. 2019;8:934. doi:10.12688/f1000research.18831.1

44. Liu X, Wang L, Ma C, et al. Exosomes derived from platelet-rich plasma present a novel potential in alleviating knee osteoarthritis by promoting proliferation and inhibiting apoptosis of chondrocyte via Wnt/3-catenin signaling pathway. J Orthop Surg Res. 2019;14(1). doi:10.1186/s13018019-1529-7

45. Fidelix TSA, Macedo CR, Maxwell LJ, Fernandes Moça Trevisani V. Diacerein for osteoarthritis. Cochrane Db Syst Rev. $2014 ;(2)$ :CD005117. doi:10.1002/14651858.CD005117.pub3

46. Fleischmann RM, Bliddal H, Blanco FJ, et al. A phaseII TRIAL of lutikizumab, an anti-interleukin- $1 \alpha / \beta$ dual variable domain immunoglobulin, in knee osteoarthritis patients with synovitis. Arthrit Rheumatol. 2019;71(7):1056-1069. doi:10.1002/art.40840

47. Wang T, He C. Pro-inflammatory cytokines: the link between obesity and osteoarthritis. Cytokine Growth F R. 2018;44:38-50. doi:10.1016/j. cytogfr.2018.10.002

48. Idriss HT, Naismith JH. TNF alpha and the TNF receptor superfamily: structure-function relationship(s). Microsc Res Tech. 2000;50(3):184-195. doi:10.1002/1097-0029(20000801)50:3<184::AID-JEMT2>3.0.CO;2-H

49. Wojdasiewicz P, Poniatowski AA, Szukiewicz D. The role of inflammatory and anti-inflammatory cytokines in the pathogenesis of osteoarthritis. Mediat Inflamm. 2014;2014:1-19. doi:10.1155/2014/561459

50. Han F, Jiang H, Qu W, Rui Y. KLF11 protects chondrocytes via inhibiting p38 MAPK signaling pathway. Eur Rev Med Pharmacol. 2020;24 (12):6505.

51. Lei J, Fu Y, Zhuang Y, Zhang K, Lu D. LncRNA SNHG1 alleviates IL-1 $\beta$-induced osteoarthritis by inhibiting miR-16-5p-mediated p38 MAPK and NF- $\kappa$ B signaling pathways. Bioscience Rep. 2019;39(9). doi:10.1042/BSR20191523

52. Cao Z, Liu W, Qu X, et al. miR-296-5p inhibits IL-1 $\beta$-induced apoptosis and cartilage degradation in human chondrocytes by directly targeting TGF- $\beta 1 /$ CTGF/p38MAPK pathway. Cell Cycle. 2020;19(12):1443-1453. doi:10.1080/15384101.2020.1750813

53. Surinkaew S, Kumphune S, Chattipakorn S, Chattipakorn N. Inhibition of p38 MAPK during ischemia, but not reperfusion, effectively attenuates fatal arrhythmia in ischemia/reperfusion heart. J Cardiovasc Pharm. 2013;61(2):133-141. doi:10.1097/FJC.0b013e318279b7b1

54. Sugiyama N, Kohno M, Yokoyama T. Inhibition of the p38 MAPK pathway ameliorates renal fibrosis in an NPHP2 mouse model. Nephrol Dial Transpl. 2012;27(4):1351-1358. doi:10.1093/ndt/gfr550

55. Zhang S, Cao H, Li Y, et al. Metabolic benefits of inhibition of p38 $\alpha$ in white adipose tissue in obesity. PLoS Biol. 2018;16(5):e2004225. doi:10.1371/journal.pbio.2004225

56. Lee JK, Kim N. Recent advances in the inhibition of p38 MAPK as a potential strategy for the treatment of Alzheimer's disease. Molecules. 2017;22(8):1287. doi:10.3390/molecules22081287

57. Wang S, Ding L, Ji H, et al. The role of p38 MAPK in the development of diabetic cardiomyopathy. Int J Mol Sci. 2016;17(7):1037. doi:10.3390/ ijms 17071037

58. Kang S, Kim J, Shin DY. Inhibition of senescence and promotion of the proliferation of chondrocytes from articular cartilage by CsA and FK506 involves inhibition of p38MAPK. Mech Ageing Dev. 2016;153:7-13. doi:10.1016/j.mad.2015.12.002

59. Zhang H, Lin J, Chen J, et al. Effects of Notch/p38MAPK signaling pathway on articular cartilage defect recovery by BMSCs tissue based on the rabbit articular cartilage defect models. Saudi J Biol Sci. 2020;27(3):859-864. doi:10.1016/j.sjbs.2019.12.021

60. Zhou Z, Tang SA, Nie X, et al. Osteoarthritic infrapatellar fat pad aggravates cartilage degradation via activation of p38MAPK and ERK1/2 pathways. Inflamm Res. 2021;70(10-12):1129-1139. doi:10.1007/s00011-021-01503-9

61. Song W, Dong Y, Luo C, Chen Y. p38MAPK family isoform p38 $\alpha$ and activating transcription factor 2 are associated with the malignant phenotypes and poor prognosis of patients with ovarian adenocarcinoma. Pathol Res Pract. 2017;213(10):1282-1288. doi:10.1016/j. prp.2017.08.003 
62. Chen X, Lv R, Zhang W, et al. Inhibition of myocyte-specific enhancer factor $2 \mathrm{~A}$ improved diabetic cardiac fibrosis partially by regulating endothelial-to-mesenchymal transition. Oncotarget. 2016;7(21):31053-31066. doi:10.18632/oncotarget.8842

63. Sugden PH, Clerk A. "Stress-responsive" mitogen-activated protein kinases (c-Jun N-terminal kinases and p38 mitogen-activated protein kinases) in the myocardium. Circ Res. 1998;83(4):345-352. doi:10.1161/01.RES.83.4.345

64. Jovanovic M, Hengartner MO. miRNAs and apoptosis: RNAs to die for. Oncogene. 2006;25(46):6176-6187. doi:10.1038/sj.onc.1209912

65. Hobert O. Gene regulation by transcription factors and microRNAs. Science. 2008;319(5871):1785-1786. doi:10.1126/science.1151651

66. Rasheed Z, Rasheed N, Abdulmonem WA, Khan MI. MicroRNA-125b-5p regulates IL-1 $\beta$ induced inflammatory genes via targeting TRAF6mediated MAPKs and NF-אB signaling in human osteoarthritic chondrocytes. Sci Rep UK. 2019;9(1):6882. doi:10.1038/s41598-019-42601-3

67. Yuan L, Chang X, Yao W, et al. MiR-214 inhibits knee osteoarthritis in rats through MAPK signaling pathway. Panminerva Med. 2019. doi:10.23736/S0031-0808.19.03716-9

68. Cuenda A, Rouse J, Doza YN, et al. SB 203580 is a specific inhibitor of a MAP kinase homologue which is stimulated by cellular stresses and interleukin-1. FEBS Lett. 1995;364(2):229-233.

69. Morel C, Ibarz G, Oiry C, et al. Cross-interactions of two p38 mitogen-activated protein (MAP) kinase inhibitors and two cholecystokinin (CCK) receptor antagonists with the CCK1 receptor and P38 MAP Kinase. J Biol Chem. 2005;280(22):21384-21393. doi:10.1074/jbc.M408851200

70. Wang G, Pang Z, Chen-Yu Hsu A, et al. Combined treatment with SB203580 and dexamethasone suppresses non-typeable Haemophilus influenzae-induced Th17 inflammation response in murine allergic asthma. Eur J Pharmacol. 2019;862:172623. doi:10.1016/j.ejphar.2019.172623

71. Jin X, Gong P, Li G, Zhang X, Li J. The p38 MAPK inhibitor, SB203580, inhibits cell invasion by Neospora caninum. Parasitol Res. 2017;116 (2):813-819. doi:10.1007/s00436-016-5346-1

72. Gao X, Li N, Zhang J. SB203580, a p38MAPK inhibitor, attenuates olfactory dysfunction by inhibiting OSN apoptosis in AR mice (activation and involvement of the p38 mitogen-activated protein kinase in olfactory sensory neuronal apoptosis of OVA-induced allergic rhinitis). Brain Behav. 2019;9(6):e01295-n/a. doi:10.1002/brb3.1295

73. Hill RJ, Dabbagh K, Phippard D, et al. Pamapimod, a novel p38 mitogen-activated protein kinase inhibitor: preclinical analysis of efficacy and selectivity. J Pharmacol Exp Ther. 2008;327(3):610-619. doi:10.1124/jpet.108.139006

74. Zhao X, Ning L, Xie Z, et al. The novel p38 inhibitor, pamapimod, inhibits osteoclastogenesis and counteracts estrogen-dependent bone loss in mice. J Bone Miner Res. 2019;34(5):911-922. doi:10.1002/jbmr.3655

75. Zhang J, Yan C, He W, Wang M, Liu J. Inhibition against p38/MEF2C pathway by Pamapimod protects osteoarthritis chondrocytes hypertrophy. Panminerva Med. 2020. doi:10.23736/S0031-0808.20.04170-1

76. Zhao C, Gao Y, Song W, et al. An update on the emerging role of resistin on the pathogenesis of osteoarthritis. Mediat Inflamm. 2019;2019:1-8.

77. Chen W, Lin C, Kuo S, et al. Resistin enhances VCAM-1 expression and monocyte adhesion in human osteoarthritis synovial fibroblasts by inhibiting miR-381 expression through the PKC, p38, and JNK signaling pathways. Cells-Basel. 2020;9(6):1369. doi:10.3390/cells9061369

78. Huang X, Ni B, Xi Y, et al. Protease-activated receptor 2 (PAR-2) antagonist AZ3451 as a novel therapeutic agent for osteoarthritis. Aging. 2019;11 (24):12532-12545. doi:10.18632/aging.102586

79. Ren C, Liang Z. Piperine alleviates lipopolysaccharide-induced inflammatory injury by down-regulating microRNA-127 in murine chondrogenic ATDC5 cells. Biomed Pharmacother. 2018;103:947-954. doi:10.1016/j.biopha.2018.04.108

80. Fan L, Li M, Cao F, et al. Astragalus polysaccharide ameliorates lipopolysaccharide-induced cell injury in ATDC5 cells via miR-92a/KLF4 mediation. Biomed Pharmacother. 2019;118:109180. doi:10.1016/j.biopha.2019.109180

81. Jin H, Zhang H, Ma T, et al. Resveratrol protects murine chondrogenic ATDC5 cells against LPS-induced inflammatory injury through up-regulating MiR-146b. Cell Physiol Biochem. 2018;47(3):972-980. doi:10.1159/000490141

82. Li Q, Xing W, Xu X, et al. Tetramethylpyrazine alleviates lipopolysaccharide-induced damage in ATDC5 cells via down-regulating MyD88. Exp Mol Pathol. 2019;111:104317. doi:10.1016/j.yexmp.2019.104317

83. Feng S, Cong H, Ji L. Salvianolic acid A exhibits anti-inflammatory and antiarthritic effects via inhibiting NF-kB and p38/MAPK pathways. Drug Des Devel Ther. 2020;14:1771-1778. doi:10.2147/DDDT.S235857

84. Li H, You Y, Jiang B, et al. Wang-Bi tablet ameliorates DMM-induced knee osteoarthritis through suppressing the activation of p38-MAPK and NF-kB signaling pathways in mice. Evid Based Compl Alt. 2021;2021:1-9. doi:10.1155/2021/2209574

85. Yao Y, Wang Y. ATDC5: an excellent in vitro model cell line for skeletal development. J Cell Biochem. 2013;114(6):1223-1229. doi:10.1002/ jcb. 24467

86. Noguchi H, Miyagi Shiohira C, Nakashima Y, Saitoh I, Watanabe M. Novel cell-permeable p38-MAPK inhibitor efficiently prevents porcine islet apoptosis and improves islet graft function. Am J Transplant. 2020;20(5):1296-1308. doi:10.1111/ajt.15740

87. Wagner SJ, Skripchenko A, Seetharaman S, Kurtz J. Amelioration of lesions associated with 24-hour suboptimal platelet storage at $16{ }^{\circ} \mathrm{C}$ by a p38MAPK inhibitor, VX-702. Vox Sang. 2015;108(3):226-232. doi:10.1111/vox.12221

88. Damjanov N, Kauffman RS, Spencer-Green GT. Efficacy, pharmacodynamics, and safety of VX-702, a novel p38 MAPK inhibitor, in rheumatoid arthritis: results of two randomized, double-blind, placebo-controlled clinical studies. Arthritis Rheum. 2009;60(5):1232-1241. doi:10.1002/ art. 24485

89. Shao B, Xu Z, Han B, Su D, Liu C. NLRP3 inflammasome and its inhibitors: a review. Front Pharmacol. 2015;6. doi:10.3389/fphar.2015.00262

90. Ni B, Pei W, Qu Y, et al. MCC950, the NLRP3 inhibitor, protects against cartilage degradation in a mouse model of osteoarthritis. Oxid Med Cell Longev. 2021;2021:1-14.

91. Wang H, Shan XB, Qiao YJ. PDK2 promotes chondrogenic differentiation of mesenchymal stem cells by upregulation of Sox6 and activation of JNK/MAPK/ERK pathway. Braz J Med Biol Res. 2017;50(2):e5988-e5988. doi:10.1590/1414-431x20165988

92. Benabdoune H, Rondon E, Shi Q, et al. The role of resolvin D1 in the regulation of inflammatory and catabolic mediators in osteoarthritis. Inflamm Res. 2016;65(8):635-645. doi:10.1007/s00011-016-0946-x

93. Zheng S, Ren J, Gong S, Qiao F, He J. CTRP9 protects against MIA-induced inflammation and knee cartilage damage by deactivating the MAPK/ NF-KB pathway in rats with osteoarthritis. Open Life Sci. 2020;15(1):971-980. doi:10.1515/biol-2020-0105

94. Ito T, Omori K, Rawson J, et al. Improvement of canine islet yield by donor pancreas infusion with a p38MAPK inhibitor. Transplantation. 2008;86 (2):321-329. doi:10.1097/TP.0b013e31817ef6c9 
95. Bagley MC, Dwyer JE, Baashen M, et al. The effect of RO3201195 and a pyrazolyl ketone P38 MAPK inhibitor library on the proliferation of Werner syndrome cells. Org Biomol Chem. 2016;14(3):947-956. doi:10.1039/C5OB02229K

\section{Publish your work in this journal}

The Journal of Inflammation Research is an international, peer-reviewed open-access journal that welcomes laboratory and clinical findings on the molecular basis, cell biology and pharmacology of inflammation including original research, reviews, symposium reports, hypothesis formation and commentaries on: acute/chronic inflammation; mediators of inflammation; cellular processes; molecular mechanisms; pharmacology and novel anti-inflammatory drugs; clinical conditions involving inflammation. The manuscript management system is completely online and includes a very quick and fair peer-review system. Visit http://www.dovepress.com/testimonials.php to read real quotes from published authors.

Submit your manuscript here: https://www.dovepress.com/journal-of-inflammation-research-journal 